\title{
Extremal Solutions for a Class of Tempered Fractional Turbulent Flow Equations in a Porous Medium
}

\author{
Xinguang Zhang $\mathbb{D}^{1,2}$ Jiqiang Jiang, ${ }^{3}$ Lishan Liu, ${ }^{3}$ and Yonghong $\mathrm{Wu}^{2}$ \\ ${ }^{1}$ School of Mathematical and Informational Sciences, Yantai University, Yantai 264005, Shandong, China \\ ${ }^{2}$ Department of Mathematics and Statistics, Curtin University of Technology, Perth 6845, WA, Australia \\ ${ }^{3}$ School of Mathematical Sciences, Qufu Normal University, Qufu 273165, Shandong, China
}

Correspondence should be addressed to Xinguang Zhang; zxg123242@163.com

Received 17 August 2020; Revised 7 September 2020; Accepted 11 September 2020; Published 25 September 2020

Academic Editor: Chris Goodrich

Copyright (c) 2020 Xinguang Zhang et al. This is an open access article distributed under the Creative Commons Attribution License, which permits unrestricted use, distribution, and reproduction in any medium, provided the original work is properly cited.

\begin{abstract}
In this paper, we are concerned with the existence of the maximum and minimum iterative solutions for a tempered fractional turbulent flow model in a porous medium with nonlocal boundary conditions. By introducing a new growth condition and developing an iterative technique, we establish new results on the existence of the maximum and minimum solutions for the considered equation; at the same time, the iterative sequences for approximating the extremal solutions are performed, and the asymptotic estimates of solutions are also derived.
\end{abstract}

\section{Introduction}

Tempered stable laws were introduced to model turbulent velocity fluctuations of physics [1]. Normally, tempered stable laws retain their signature power-law behaviour at infinity and infinite divisibility [2]. By multiplying by an exponential factor for the usual second derivative, one can obtain tempered fractional derivatives and integrals. In [3], an exponential tempering factor was applied to the particle jump density in random walk and stochastic model for turbulence in the inertial range, which is the fractional derivative of Brownian motion exhibiting semilong range dependence with a power law at moderate time scales.

Tempered stable laws are useful in statistical physics and provide a basic physical model such as turbulent flow for the underlying physical phenomena. Motivated by these physical backgrounds and the sources, in this paper, we focus on the existence of the maximum and minimum iterative solutions for the following tempered fractional turbulent flow equation with nonlocal boundary conditions:

$$
\left\{\begin{array}{l}
{ }_{0}^{R} \mathbb{D}_{t}^{\alpha, \lambda}\left(\varphi_{p}\left({ }_{0}^{R} \mathbb{D}_{t}^{\beta, \lambda} x(t)\right)\right)=h(t) f(x(t)), \\
x(0)=x^{\prime}(0)=\cdots=x^{(n-2)}(0)={ }_{0}^{R} \mathbb{D}_{t}^{\beta, \lambda} x(0)=0, \\
{ }_{0}^{R} \mathbb{D}_{t}^{\beta, \lambda} x(1)=\int_{0}^{1} e^{-\lambda(1-t)} x(t) \mathrm{d} t, \\
{ }_{0}^{R} \mathbb{D}_{t}^{\gamma, \lambda}\left(\varphi_{p}\left({ }_{0}^{R} \mathbb{D}_{t}^{\beta, \lambda} x\right)\right)(1)=\int_{0}^{1} a(t)_{0}^{R} \mathbb{D}^{\gamma, \lambda}\left(\varphi_{p}\left({ }_{0}^{R} \mathbb{D}_{t}^{\beta, \lambda} x(t)\right)\right) \mathrm{d} A(t),
\end{array}\right.
$$

where $1<\alpha \leq 2, n-1<\beta \leq n, n \geq 4,0<\gamma<\alpha-1, \lambda>0$ is a constant, $\varphi_{p}$ is the $p$-Laplacian operator defined by $\varphi_{p}(s)=|s|^{p-2} s, p>1,{ }_{0}^{R} \mathbb{D}_{t}^{\alpha, \lambda}$ is the tempered fractional derivative, $\int_{0}^{1} a(t)_{0}^{R} \mathbb{D}_{t}^{\gamma, \lambda}\left(\varphi_{p}\left({ }_{0}^{R} \mathbb{D}_{t}^{\beta, \lambda} x(t)\right)\right) \mathrm{d} A(t)$ denotes a Riemann-Stieltjes integral, $A$ is a function of bounded variation, $f:[0,+\infty) \longrightarrow(0,+\infty)$ is continuous, and $h \in[L(0,1),[0,+\infty)]$.

Turbulent flow is a fundamental fluid mechanics problem which can be described by a $p$-Laplacian equation with a suitable boundary condition; for details, see [4]. Particularly, if the model is of fractional order, then it 
can describe turbulent flow in a porous medium [5-10]. On the contrary, fractional-order derivative has nonlocal characteristics; based on this property, the fractional differential equation can also interpret many abnormal phenomena that occur in applied science and engineering, such as viscoelastic dynamical phenomena [11-29], advection-dispersion process in anomalous diffusion [30-34], and bioprocesses with genetic attribute $[35,36]$. As a powerful tool of modeling the above phenomena, in recent years, the fractional calculus theory has been perfected gradually by many researchers, and various different types of fractional derivatives were studied, such as Riemann-Liouville derivatives [16, 37-62], Hadamardtype derivatives [63-71], Katugampola-Caputo derivatives [72], conformable derivatives [73-76], Caputo-Fabrizio derivatives [77, 78], Hilfer derivatives [79-82], and tempered fractional derivatives [83]. These works also enlarged and enriched the application of the fractional calculus in impulsive theories [84-89], chaotic system [90-93], and resonance phenomena [94-96]. Among them, by using the fixed point theorem of the mixed monotone operator, Zhang et al. [9] established the result of uniqueness of the positive solution for the Riemann-Liouville-type turbulent flow in a porous medium:

$$
\left\{\begin{array}{l}
\mathscr{D}_{\mathbf{t}}^{\beta}\left(\varphi_{p}\left(-\mathscr{D}_{\mathbf{t}}^{\alpha} z\right)\right)(t)=-f\left(z(t), \mathscr{D}_{\mathbf{t}}^{\gamma} z(t)\right), \quad t \in(0,1), \\
\mathscr{D}_{\mathbf{t}}^{\alpha} z(0)=\mathscr{D}_{\mathbf{t}}^{\alpha+1} z(0)=\mathscr{D}_{\mathbf{t}}^{\alpha} z(1)=0, \\
\mathscr{D}_{\mathbf{t}}^{\gamma} z(0)=0, \mathscr{D}_{\mathbf{t}}^{\gamma} z(1)=\int_{0}^{1} \mathscr{D}_{\mathbf{t}}^{\gamma} z(s) \mathrm{d} A(s),
\end{array}\right.
$$

where $0<\gamma \leq 1<\alpha \leq 2<\beta<3, \alpha-\gamma>1, \quad \mathscr{D}_{\mathbf{t}}^{\alpha}$, $\mathscr{D}_{\mathbf{t}}^{\beta}$, and $\mathscr{D}_{\mathbf{t}}^{\gamma}$ denote the Riemann-Liouville derivatives, and $\int_{0}^{1} z(s) \mathrm{d} A(s)$ indicates the Riemann-Stieltjes integral, and $A$ is a function of bounded variation; the nonlinear term may be singular at both first variable and second variable. Recently, Zhou et al. [83] investigated a class of tempered fractional differential equations with Riemann-Stieltjes integral boundary conditions; by using the fixed point theorem of the sum-type mixed monotone operator, the existence and uniqueness of positive solutions were established, and iterative sequences for approximating the unique positive solution were also constructed.

However, to the best of our knowledge, there are relatively few results on fractional turbulent flow in a porous medium with nonlocal Riemann-Stieltjes integral boundary conditions, and no work has been reported on the maximal and minimal solutions for the tempered-type fractional turbulent flow equation. Thus, following the previous work, this paper will pay attention to the extremal solutions for the tempered fractional turbulent flow equation in a porous medium with nonlocal Riemann-Stieltjes integral boundary conditions by developing iterative technique, also see [97-100]. Different from [9, 83], in this paper, we will give a new type of growth condition for the nonlinear term to guarantee equation (1) has the extremal solutions. At the same time, the iterative sequences for approximating the extremal solutions are performed, and the asymptotic estimates of solutions are also obtained.

\section{Preliminaries and Lemmas}

Before starting our work, we firstly recall the definition of the tempered fractional derivative which is an extension of the Riemann-Liouville derivative and integral.

Let $\lambda>0$; the $\alpha$-order left tempered fractional derivative is defined by

$$
{ }_{0}^{R} \mathbb{D}_{\mathbf{t}}^{\alpha, \lambda} x(t)=e^{-\lambda t R} \mathscr{D}_{\mathbf{t}}^{\alpha}\left(e^{\lambda t} x(t)\right)
$$

where ${ }_{0}^{R} \mathscr{D}_{\mathrm{t}}^{\alpha}$ denotes the standard Riemann-Liouville fractional derivative which can be found in [101].

Let

$$
\begin{aligned}
H(t, s) & =\left\{\begin{array}{l}
\frac{\left[\beta(1-s)^{\beta-1}(\beta-1+s) e^{\lambda s} t^{\beta-1}-\beta(\beta-1) e^{\lambda s}(t-s)^{\beta-1}\right] e^{-\lambda t}}{(\beta-1) \Gamma(\beta+1)}, \quad 0 \leq s \leq t \leq 1, \\
\frac{\left[\beta(1-s)^{\beta-1}(\beta-1+s)\right] e^{\lambda s}}{(\beta-1) \Gamma(\beta+1)} t^{\beta-1} e^{-\lambda t}, \quad 0 \leq t \leq s \leq 1,
\end{array}\right. \\
\Delta & =\frac{e^{-\lambda}-\delta}{\Gamma(\alpha-\gamma)} \\
\delta & =\int_{0}^{1} e^{-\lambda s} s^{\alpha-\gamma-1} a(s) \mathrm{d} A(s) .
\end{aligned}
$$

The following results have been proven in [83]. 
Lemma 1. Given $k \in C[0,1]$; then, the boundary value problem,

$$
\left\{\begin{array}{l}
{ }_{0}^{R} \mathbb{D}_{t}^{\alpha, \lambda}\left(\varphi_{p}\left({ }_{0}^{R} \mathbb{D}_{t}^{\beta, \lambda} x(t)\right)\right)=k(t), \\
x(0)=x^{\prime}(0)=\cdots=x^{(n-2)}(0)={ }_{0}^{R} \mathbb{D}_{t}^{\beta, \lambda} x(0)=0, \\
{ }_{0}^{R} \mathbb{D}_{t}^{\beta, \lambda} x(1)=\int_{0}^{1} e^{-\lambda(1-t)} x(t) \mathrm{d} t, \\
{ }_{0}^{R} \mathbb{D}_{t}^{\gamma, \lambda}\left(\varphi_{p}\left({ }_{0}^{R} \mathbb{D}_{t}^{\beta, \lambda} x\right)\right)(1)=\int_{0}^{1} a(t){ }_{0}^{R} \mathbb{D}_{t}^{\gamma, \lambda}\left(\varphi_{p}\left({ }_{0}^{R} \mathbb{D}_{t}^{\beta, \lambda} x(t)\right)\right) \mathrm{d} A(t),
\end{array}\right.
$$

has the unique solution

$$
x(t)=\int_{0}^{1} H(t, s) \varphi_{q}\left(\int_{0}^{1} G(t, \tau) k(\tau) \mathrm{d} \tau\right) \mathrm{d} s,
$$

where $H(t, s)$ is defined by (4) and $G(t, s)$ denotes the Green function as follows:

$$
\begin{aligned}
& G(t, s)=G_{1}(t, s)+\frac{t^{\alpha-1} e^{-\lambda t}}{\Delta \Gamma(\alpha-\gamma)} \int_{0}^{1} a(t) G_{2}(t, s) \mathrm{d} A(t), \\
& G_{1}(t, s)=\frac{e^{\lambda(s-t)}}{\Gamma(\alpha)} \begin{cases}(1-s)^{\alpha-\gamma-1} t^{\alpha-1}-(t-s)^{\alpha-1}, & 0 \leq s \leq t \leq 1, \\
(1-s)^{\alpha-2} t^{\alpha-1}, & 0 \leq t \leq s \leq 1,\end{cases} \\
& G_{2}(t, s)=\frac{e^{\lambda(s-t)}}{\Gamma(\alpha)} \begin{cases}(1-s)^{\alpha-\gamma-1} t^{\alpha-\gamma-1}-(t-s)^{\alpha-\gamma-1}, & 0 \leq s \leq t \leq 1, \\
(1-s)^{\alpha-\gamma-1} t^{\alpha-\gamma-1}, & 0 \leq t \leq s \leq 1 .\end{cases}
\end{aligned}
$$

In order to obtain the positive extremal solutions of tempered fractional turbulent flow equation (1), it is necessary to preserve nonnegativity of the Green function.

(H0):

$$
\int_{0}^{1} e^{-\lambda s} s^{\alpha-\gamma-1} a(s) \mathrm{d} A(s)<e^{-\lambda}
$$

Lemma 2. Assume (H0) holds; then, functions $G(t, s)$ and $H(t, s)$ have the following properties:

(1) $G(t, s)$ and $H(t, s)$ are nonnegative and continuous for $(t, s) \in[0,1] \times[0,1]$.

(2) For any $t, s \in[0,1], H(t, s)$ satisfies

$$
m_{1}(s) e^{-\lambda t} t^{\beta-1} \leq H(t, s) \leq M_{1}(s) e^{-\lambda t} t^{\beta-1}
$$

where

$$
\begin{aligned}
& M_{1}(s)=\frac{\beta(1-s)^{\beta-1}(\beta-1+s) e^{\lambda s}}{(\beta-1) \Gamma(\beta+1)}, \\
& m_{1}(s)=\frac{\beta s(1-s)^{\beta-1} e^{\lambda s}}{(\beta-1) \Gamma(\beta+1)}
\end{aligned}
$$

$$
m_{2}(s) e^{-\lambda t} t^{\alpha-1} \leq G(t, s) \leq M_{2}(s) e^{-\lambda t} t^{\alpha-1}, \quad s, t \in[0,1]
$$

where

$$
\begin{aligned}
& M_{2}(s)=\left[\frac{1}{\Gamma(\alpha)}+\frac{\delta}{\Delta \Gamma(\alpha) \Gamma(\alpha-\gamma)}\right] e^{\lambda s}(1-s)^{\alpha-\gamma-1}, \\
& m_{2}(s)=\frac{e^{\lambda s}\left[(1-s)^{\alpha-\gamma-1}-(1-s)^{\alpha-1}\right]}{\Gamma(\alpha)} .
\end{aligned}
$$

Let

$$
M_{1}^{*}=\frac{\beta^{2} e^{\lambda}}{(\beta-1) \Gamma(\beta+1)} .
$$

In order to obtain the existence of positive extremal solutions of tempered fractional turbulent flow equation (1), we introduce the following new control conditions.

(H1): $f:[0,+\infty) \longrightarrow(0,+\infty)$ is continuous and nondecreasing, and there exists a positive constant $\epsilon>3 /(q-1)$ such that

$$
0<d=: \sup _{s \geq 0} \frac{f(s)}{(s+2)^{\epsilon}}<+\infty .
$$

(H2):

$$
0<\int_{0}^{1} M_{2}(\tau) h(\tau) \mathrm{d} \tau<\left(\frac{M_{1}^{*}}{3^{\epsilon(q-1)}}\right)^{1 / q-1} d^{-1} .
$$

Remark 1. Assumption (14) we introduced is a new type of growth condition, which includes a large number of basic functions such as

(1) $f(t)=b_{0}+\sum_{i=1}^{n} b_{i}(t+2)^{\mu_{i}}$, where $\quad b_{0}>0, b_{i}, \mu_{i}>$ $3 / q-1, i=1,2, \ldots, n$.

(2) $f(t)=b_{0}+\left[\sum_{i=1}^{n} b_{i}(t+2)^{\mu_{i}}\right]^{1 / \mu}$, where $b_{0}>0, \mu>0$, $b_{i}>0, \mu_{i}>0(i=1,2, \ldots, n)$ and

$$
\min _{i=1, \ldots, n}\left\{\mu_{i}\right\}>\frac{3 \mu}{q-1}
$$

(3) $f(t)=(t+1)^{\mu-1} \ln (1+(1 / 2+t))+(2+t)^{\mu-2}+b$, $b>0, \mu>2+(3 / q-1)$.

(4) $f:[0,+\infty) \longrightarrow(0,+\infty)$ is continuous and nondecreasing, and there exists a positive constant $\epsilon>3 / q-1$ such that $f(x) /(x+2)^{\epsilon}$ is increasing on $x$, and

$$
\lim _{x \rightarrow+\infty} \frac{f(x)}{(x+2)^{\epsilon}}=M>0 .
$$


(5) $f:[0,+\infty) \longrightarrow(0,+\infty)$ is continuous and nondecreasing, and there exists a positive constant $\epsilon>3 / q-1$ such that $f(x) /(x+2)^{\epsilon}$ is nonincreasing on $x$.

Proof. For cases (1)-(3), take

$$
\begin{aligned}
& \epsilon=\max _{i=1,2, \ldots, n}\left\{\mu_{i}\right\}, \\
& \epsilon=\frac{\max _{i=1,2, \ldots, n}\left\{\mu_{i}\right\}}{\mu}, \\
& \epsilon=\mu-2,
\end{aligned}
$$

respectively; obviously,

$$
0<d=: \sup _{s \geq 0} \frac{f(s)}{(s+2)^{\epsilon}}<+\infty .
$$

For cases (4) and (5), it is clear; we omit the proof.

Denote $E=C[0,1]$ as all continuous functions equipped the maximum norm

$$
\|x\|=\max \{x(t): t \in[0,1]\} .
$$

Define a cone $P$,

$P=\left\{x \in E\right.$ : there exists a number $0<l_{x}<1$ such that

$$
\left.0 \leq x(t) \leq l_{x}^{-1} e^{-\lambda t} t^{\beta-1}, \quad t \in[0,1]\right\}
$$

and an operator $T$ in $E$ :

$$
T(x)(t)=\int_{0}^{1} H(t, s) \varphi_{q}\left(\int_{0}^{1} G(s, \tau) h(\tau) f(x(\tau)) \mathrm{d} \tau\right) \mathrm{d} s .
$$

Then, the fixed point of operator $T$ in $E$ is the solution of tempered fractional turbulent flow equation (1).

Lemma 3. Assume that $(\mathbf{H} 0)-(\mathbf{H} 2)$ hold. Then, $T: P \longrightarrow P$ is a continuous, compact operator.

Proof. It follows from the definition of $P$ that, for any $x \in P$, there exists a number $0<l_{x}<1$ such that

$$
0 \leq x(t) \leq l_{x}^{-1} e^{-\lambda t} t^{\beta-1}, \quad t \in[0,1] .
$$

Since $T$ is increasing with respect to $x$, by (14), (23), and Lemma 2, we have

$$
\begin{aligned}
T(x)(t) & =\int_{0}^{1} H(t, s) \varphi_{q}\left(\int_{0}^{1} G(s, \tau) h(\tau) f(x(\tau)) \mathrm{d} \tau\right) \mathrm{d} s \\
& \leq e^{-\lambda t} t^{\beta-1} \int_{0}^{1} M_{1}(s) \varphi_{q}\left(\int_{0}^{1} G(s, \tau) h(\tau) f(x(\tau)) \mathrm{d} \tau\right) \mathrm{d} s \\
& \leq e^{-\lambda t} t^{\beta-1} \int_{0}^{1} M_{1}(s) \varphi_{q}\left(\int_{0}^{1} G(s, \tau) h(\tau) \frac{f(x(\tau))}{(x(\tau)+2)^{\epsilon}}(x(\tau)+2)^{\epsilon} \mathrm{d} \tau\right) \mathrm{d} s \\
& \leq \varphi_{q}(d) e^{-\lambda t} t^{\beta-1} \int_{0}^{1} M_{1}(s) \varphi_{q}\left(\int_{0}^{1} G(s, \tau) h(\tau)(x(\tau)+2)^{\epsilon} \mathrm{d} \tau\right) \mathrm{d} s \\
& \leq \varphi_{q}(d) e^{-\lambda t} t^{\beta-1} \int_{0}^{1} M_{1}(s) \varphi_{q}\left(\int_{0}^{1} G(s, \tau) h(\tau)\left(l_{x}^{-1} e^{-\lambda \tau} \tau^{\beta-1}+2\right)^{\epsilon} \mathrm{d} \tau\right) \mathrm{d} s \\
& \leq M_{1}^{*} \varphi_{q}\left(d\left(l_{x}^{-1}+2\right)^{\epsilon} \int_{0}^{1} M_{2}(\tau) h(\tau) d \tau\right) e^{-\lambda t} t^{\beta-1} \\
& \leq \frac{1}{l_{x}^{*} e^{-\lambda t} t^{\beta-1}},
\end{aligned}
$$

where

$$
l_{x}^{*}=\min \left\{\frac{1}{2}, M_{1}^{*-1}\left(d\left(l_{x}^{-1}+2\right)^{\epsilon} \int_{0}^{1} M_{2}(\tau) h(\tau) \mathrm{d} \tau\right)^{1-q}\right\} .
$$

Thus, it follows from (24) that

$$
0 \leq T(x)(t) \leq \frac{1}{l_{x}^{*}} e^{-\lambda t} t^{\beta-1},
$$

which implies that $T$ is well defined and uniformly bounded, and $T(P) \subset P$.

On the contrary, according to the Arzela-Ascoli theorem and the Lebesgue dominated convergence theorem, it is easy to know that $T: P \longrightarrow P$ is completely continuous. 


\section{Main Results}

Before we begin to state our main result, we first give the following lemma.

Lemma 4. Suppose $\epsilon(q-1)>3$ and $(\mathbf{H} 2)$ hold; then, the equation

$$
M_{1}^{*}\left(d \int_{0}^{1} M_{2}(\tau) h(\tau) \mathrm{d} \tau\right)^{q-1}(x+3)^{\epsilon(q-1)}(x+1)^{-1}=1,
$$

has unique solution $\delta^{*}$ in $(0, \infty)$.

Proof. Let

$$
\phi(x)=1-M_{1}^{*}\left(d \int_{0}^{1} M_{2}(\tau) h(\tau) \mathrm{d} \tau\right)^{q-1}(x+3)^{\epsilon(q-1)}(x+1)^{-1} .
$$

It follows from $\epsilon(q-1)>3$ and $(\mathbf{H} 2)$ that

$$
\phi(0)=1-M_{1}^{*}\left(d \int_{0}^{1} M_{2}(\tau) h(\tau) \mathrm{d} \tau\right)^{q-1} 3^{\epsilon(q-1)}>0
$$

$\phi(+\infty)=-\infty$

On the contrary, $\phi(x)$ is a continuous function in $[0, \infty)$ satisfying

$$
\begin{aligned}
\phi^{\prime}(x) & =-\epsilon(q-1) M_{1}^{*}\left(d \int_{0}^{1} M_{2}(\tau) h(\tau) \mathrm{d} \tau\right)^{q-1}(x+3)^{\epsilon(q-1)-1}(x+1)^{-1}+M_{1}^{*}\left(d \int_{0}^{1} M_{2}(\tau) h(\tau) \mathrm{d} \tau\right)^{q-1}(x+3)^{\epsilon(q-1)}(x+1)^{-2} \\
& =-M_{1}^{*}\left(d \int_{0}^{1} M_{2}(\tau) h(\tau) \mathrm{d} \tau\right)^{q-1}(x+3)^{\epsilon(q-1)-1}(x+1)^{-1}\left(\epsilon(q-1)-\frac{x+3}{x+1}\right)<0, \quad x \in(0, \infty) .
\end{aligned}
$$

Thus, (29)-(31) imply equation (27) has unique zero point $\delta^{*}$ in $(0, \infty)$.

Theorem 1. Suppose (H0)-(H2) hold. Then, the following is obtained:

(i) Existence: equation (1) has a positive minimal solution $\underline{x}$ and a positive maximal solution $\bar{x}$.

(ii) Asymptotic estimates: there exist positive numbers $n_{i}>0, i=1,2$, such that

$$
\begin{aligned}
& \frac{\underline{x}(t)}{e^{-\lambda t} t^{\beta-1}} \in\left[0, n_{1}\right], \\
& \frac{\bar{x}(t)}{e^{-\lambda t} t^{\beta-1}} \in\left[0, n_{2}\right], t \in(0,1] .
\end{aligned}
$$

(iii) Iterative sequences: for initial values $x^{(0)}(t)=0$ and $y^{(0)}(t)=\delta^{*}+1$, construct the iterative sequences

$$
\begin{aligned}
& x^{(n)}(t)=\int_{0}^{1} H(t, s) \varphi_{q}\left(\int_{0}^{1} G(s, \tau) h(\tau) f\left(x^{(n-1)}(\tau)\right) \mathrm{d} \tau\right) \mathrm{d} s, \\
& y^{(n)}(t)=\int_{0}^{1} H(t, s) \varphi_{q}\left(\int_{0}^{1} G(s, \tau) h(\tau) f\left(y^{(n-1)}(\tau)\right) \mathrm{d} \tau\right) \mathrm{d} s .
\end{aligned}
$$

Then,

$$
\begin{aligned}
& \lim _{n \longrightarrow+\infty} x^{(n)}(t)=\underline{x}(t), \\
& \lim _{n \longrightarrow+\infty} y^{(n)}(t)=\bar{x}(t),
\end{aligned}
$$

uniformly, for $t \in[0,1]$, where $\delta^{*}$ is the unique solution of equation $(27)$ in $(0, \infty)$.
Proof. Firstly, let $P_{\delta^{*}}=\left\{x \in P: 0 \leq\|x\| \leq \delta^{*}+1\right\}$; we shall show $T\left(P_{\delta^{*}}\right) \subset P_{\delta^{*}}$.

For any $x \in P_{\delta^{*}}$ and for any $t \in(0,1)$, we have

$$
0 \leq x(t) \leq \max _{t \in[0,1]} x(t) \leq \delta^{*}+1 .
$$

Consequently, it follows from (H1) and Lemma 4 that

$$
\begin{aligned}
\|T(x)\| & =\max _{t \in[0,1]}\left\{\int_{0}^{1} H(t, s) \varphi_{q}\left(\int_{0}^{1} G(s, \tau) h(\tau) f(x(\tau)) \mathrm{d} \tau\right) \mathrm{d} s\right\} \\
& \leq \int_{0}^{1} M_{1}(s) \varphi_{q}\left(\int_{0}^{1} G(s, \tau) h(\tau) f(x(\tau)) \mathrm{d} \tau\right) \mathrm{d} s \\
& \leq \int_{0}^{1} M_{1}(s) \varphi_{q}\left(\int_{0}^{1} G(s, \tau) h(\tau) \frac{f(x(\tau))}{(x(\tau)+2)^{\epsilon}}(x(\tau)+2)^{\epsilon} \mathrm{d} \tau\right) \mathrm{d} s \\
& \leq \varphi_{q}(d) \int_{0}^{1} M_{1}(s) \varphi_{q}\left(\int_{0}^{1} G(s, \tau) h(\tau)(x(\tau)+2)^{\epsilon} \mathrm{d} \tau\right) \mathrm{d} s \\
& \leq \varphi_{q}(d) \int_{0}^{1} M_{1}(s) \varphi_{q}\left(\int_{0}^{1} G(s, \tau) h(\tau)\left(\delta^{*}+3\right)^{\epsilon} \mathrm{d} \tau\right) \mathrm{d} s \\
& \leq M_{1}^{*}\left(d \int_{0}^{1} M_{2}(\tau) h(\tau) \mathrm{d} \tau\right)^{q-1}\left(\delta^{*}+3\right)^{\epsilon(q-1)} \\
& =\delta^{*}+1,
\end{aligned}
$$

which implies that $T\left(P_{\delta^{*}}\right) \subset P_{\delta^{*}}$.

Next, take the initial value $x^{(0)}(t)=0$, and let

$$
x^{(1)}(t)=T\left(x^{(0)}\right)(t)=T(0)(t), \quad t \in[0,1] .
$$

It follows from $x^{(0)}(t) \in P_{\delta^{*}}$ that $x^{(1)}(t) \in T\left(P_{\delta^{*}}\right) \subset P_{\delta^{*}}$. Denote

$x^{(n+1)}(t)=T x^{(n)}(t)=T^{n+1} x^{(0)}(t), \quad n=1,2, \ldots$ 
By $T\left(P_{\delta^{*}}\right) \subset P_{\delta^{*}}$, we have $x_{n} \in P_{\delta^{*}}$ for $n \geq 1$. It follows from the fact of $T$ being a compact operator that $\left\{x^{(n)}\right\}$ is a sequentially compact set.

On the contrary, since $x^{(1)}(t) \geq 0=x^{(0)}(t)$ and $T$ is increasing on $x$, we have

$$
x^{(2)}(t)=\left(T x^{(1)}\right)(t) \geq\left(T x^{(0)}\right)(t)=x^{(1)}(t), \quad t \in[0,1] .
$$

By induction, one has

$$
0 \leq x^{(n)}(t) \leq x^{(n+1)}(t) \leq \delta^{*}+1, \quad n=1,2, \ldots
$$

Consequently, there exists $\underline{x} \in P_{\delta^{*}}$ such that $x^{(n)} \longrightarrow \underline{x}$. Noticing that $T x^{(n)}=x^{(n-1)}$ and letting $n \longrightarrow+\infty$, by the continuity of $T$, we have $T \underline{x}=\underline{x}$, which implies that $\underline{x}$ is a nonnegative solution of equation (1), and then $\underline{x}$ is a positive solution of equation (1) since $f(0)>0$.

Now, we take $y^{(0)}(t)=\delta^{*}+1$ as the initial value and let

$$
y^{(1)}(t)=\left(T\left(y^{(0)}\right)(t)\right), \quad t \in[0,1] .
$$

It follows from $y^{(0)}(t)=\delta^{*}+1 \in P_{\delta^{*}}$ that $y^{(1)} \in P_{\delta^{*}}$. Thus, construct the iterative sequence

$$
y^{(n+1)}(t)=T y^{(n)}(t)=T^{n+1} y^{(0)}(t), \quad n=1,2, \ldots .
$$

We have

$$
y^{(n)}(t) \in P_{\delta *}, \quad n=0,1,2, \ldots,
$$

since $T\left(P_{\delta^{*}}\right) \subset P_{\delta^{*}}$. It follows from Lemma 3 that $\left\{y^{(n)}\right\}$ is a sequentially compact set.

Now, since $y^{(1)} \in P_{\delta^{*}}$ and $T$ is increasing, one has

$$
0 \leq y^{(1)}(t) \leq\left\|y^{(1)}\right\| \leq \delta^{*}+1=y^{(0)}(t),
$$

and then

$$
y^{(2)}(t)=T y^{(1)}(t) \leq T y^{(0)(t)}=y^{(1)}(t) .
$$

It follows from induction that

$$
0 \leq y^{(n+1)}(t) \leq y^{(n)}(t) \leq \delta^{*}+1, \quad n=0,1,2, \ldots,
$$

which implies that there exists $\bar{x} \in P_{\delta^{*}}$ such that $y^{(n)} \longrightarrow \bar{x}$. Letting $n \longrightarrow+\infty$, from the continuity of $T$ and $T y^{(n)}=y^{(n-1)}$, we have $T \bar{x}=\bar{x}$, which implies that $\bar{x}$ is another positive solution of equation (1).

Next, we prove that $\underline{x}$ and $\bar{x}$ are the maximum and minimum positive solutions of equation (1). In fact, suppose $\tilde{x}$ is any positive solution of equation (1); then, we have

$$
\begin{aligned}
x^{(0)}(t) & =0 \leq \tilde{x}(t) \leq \delta^{*}+1=y^{(0)}(t), \\
x^{(1)}(t) & =T x^{(0)}(t) \leq T \tilde{x}(t)=\tilde{x}(t) \leq T\left(y^{(0)}\right)(t)=y^{(1)}(t) .
\end{aligned}
$$

Thus, it follows from induction that

$$
x^{(n)}(t) \leq \tilde{x}(t) \leq y^{(n)}(t), \quad n=1,2,3, \ldots
$$

Taking the limit, we have

$$
\underline{x} \leq \tilde{x} \leq \bar{x},
$$

which implies that $x$ and $\bar{x}$ are the maximal and minimal positive solutions of equation (1), respectively.

In the end, since $\underline{x}, \bar{x} \in P_{\delta^{*}} \subset P$, there exist constants $n_{1}>, n_{2}>0$ such that

$$
\begin{aligned}
& \frac{\underline{x}(t)}{e^{-\lambda t} t^{\beta-1}} \in\left[0, n_{1}\right], \\
& \frac{\bar{x}(t)}{e^{-\lambda t} t^{\beta-1}} \in\left[0, n_{2}\right], \quad t \in(0,1] .
\end{aligned}
$$

\section{Example}

Since the fractional-order derivative possesses long-memory characteristics, in fluid mechanics, equation (1) can describe a turbulent flow in a porous medium. Here, we give a specific example to illustrate the main results.

Example: consider the following nonlocal tempered fractional turbulent flow equation:

$$
\left\{\begin{array}{l}
{ }_{0}^{R} \mathbb{D}_{t}^{(3 / 2), 1}\left(\varphi_{(3 / 2)}\left({ }_{0}^{R} \mathbb{D}_{t}^{(7 / 2), 1} x(t)\right)\right)=\frac{e^{-t}(1-t)^{-(1 / 4)}}{400}\left((x(t)+1)^{3} \ln \left(1+\frac{1}{2+x(t)}\right)+(2+x(t))^{2}+2\right), \\
x(0)=x^{\prime}(0)=x^{\prime \prime}(0)={ }_{0}^{R} \mathbb{D}_{t}^{(7 / 2), 1} x(0)=0, \\
{ }_{0}^{R} \mathbb{D}_{t}^{(7 / 2), 1} x(1)=\int_{0}^{1} e^{-(1-t)} x(t) \mathrm{d} t, \\
{ }_{0}^{R} \mathbb{D}_{t}^{(1 / 4), 1}\left(\varphi_{(3 / 2)}\left({ }_{0}^{R} \mathbb{D}_{t}^{(7 / 2), 1} x\right)\right)(1)=\int_{0}^{1}{ }_{0}^{R} \mathbb{D}_{t}^{(1 / 4), 1}\left(\varphi_{(3 / 2)}\left({ }_{0}^{R} \mathbb{D}_{t}^{(7 / 2), 1} x(t)\right)\right) \mathrm{d} A(t),
\end{array}\right.
$$

where 


$$
A(t)= \begin{cases}0, & t \in\left[0, \frac{1}{2}\right), \\ 1, & t \in\left[\frac{1}{2}, \frac{3}{4}\right), \\ \frac{1}{2}, & t \in\left[\frac{3}{4}, 1\right] .\end{cases}
$$

Then, equation (51) has the positive minimal and maximal solutions $\underline{x}$ and $\bar{x}$, and there exist constants $n_{1}>, n_{2}>0$ such that

$$
\begin{aligned}
& \frac{\underline{x}(t)}{e^{-t} t^{5 / 2}} \in\left[0, n_{1}\right], \\
& \frac{\bar{x}(t)}{e^{-t} t^{5 / 2}} \in\left[0, n_{2}\right],
\end{aligned}
$$

$t \in(0,1]$.

Let

$$
\begin{aligned}
\alpha & =\frac{3}{2}, \\
\beta & =\frac{7}{2}, \\
\lambda & =1, \\
\gamma & =\frac{1}{4}, \\
p & =\frac{3}{2}, \\
a(t) & =1, \\
h(t) & =\frac{1}{400} e^{-t}(1-t)^{-1 / 4}, \\
f(x) & =(x+1)^{3} \ln \left(1+\frac{1}{2+x}\right)+(2+x)^{2}+2 .
\end{aligned}
$$

Firstly, we have

$$
\begin{aligned}
\delta & =\int_{0}^{1} e^{-\lambda s} s^{\alpha-\gamma-1} a(s) \mathrm{d} A(s)=\int_{0}^{1} e^{-s} s^{1 / 4} \mathrm{~d} A(s) \\
& =0.2902<e^{-1}=0.3679 .
\end{aligned}
$$

Thus, (H0) holds.

Obviously, $f:[0,+\infty) \longrightarrow(0,+\infty)$ is continuous and nondecreasing. Take $\epsilon=2>3 / q-1=3 / 2$; then, we have

$$
\begin{aligned}
0<d & =\sup _{x \geq 0} \frac{f(x)}{(x+2)^{\epsilon}}=\sup _{x \geq 0}\left(\left(\frac{x+1}{x+2}\right)^{3} \ln \left(1+\frac{1}{2+x}\right)^{x+2}\right. \\
& \left.+1+\frac{2}{(x+2)^{2}}\right)=2<+\infty,
\end{aligned}
$$

which implies that $(\mathbf{H} 1)$ is satisfied.

Now, we compute $M_{1}^{*}$ and $\Delta$ :

$$
\begin{aligned}
M_{1}^{*} & =\frac{\beta^{2} e^{\lambda}}{(\beta-1) \Gamma(\beta+1)}=\frac{3.5^{2} \times e}{2.5 \times \Gamma(4.5)}=0.1550, \\
\Delta & =\frac{e^{-\lambda}-\delta}{\Gamma(\alpha-\gamma)}=\frac{e^{-1}-0.3679}{\Gamma(5 / 4)}=0.0857 .
\end{aligned}
$$

Thus, we have

$$
\begin{aligned}
M_{2}(s) & =\left[\frac{1}{\Gamma(3 / 2)}+\frac{0.3679}{0.0857 \times \Gamma(3 / 2) \Gamma(5 / 4)}\right] e^{s}(1-s)^{1 / 4} \\
& =5.3439 e^{s}(1-s)^{1 / 4} .
\end{aligned}
$$

Consequently,

$$
0<\int_{0}^{1} M_{2}(\tau) h(\tau) \mathrm{d} \tau=0.013358<\left(\frac{M_{1}^{*}}{3^{\epsilon(q-1)}}\right)^{1 / q-1} d^{-1}=0.0219 .
$$

So, condition (H3) holds.

Thus, by Theorem 1, equation (51) has a positive minimal solution $\underline{x}$ and a positive maximal solution $\bar{x}$, and there exist constants $n_{1}>, n_{2}>0$ such that

$$
\begin{aligned}
& \frac{\underline{x}(t)}{e^{-t} t^{5 / 2}} \in\left[0, n_{1}\right], \\
& \frac{\bar{x}(t)}{e^{-t} t^{5 / 2}} \in\left[0, n_{2}\right],
\end{aligned}
$$

$$
t \in(0,1]
$$

\section{Conclusion}

In this work, we establish a new result on the existence of the maximum and minimum solutions for a class of tempered fractional-order differential equations with nonlocal boundary conditions. This type of equation can describe a turbulent flow of a porous medium in fluid mechanics and diffusive interaction. In order to obtain the extremal solutions of the equation, a new type of growth condition is introduced, and the iterative sequences with explicit initial values are constructed which converge uniformly to the maximum and minimum solutions; in addition, the estimations of the upper bounds of the maximum and minimum solutions are also derived. 


\section{Data Availability}

No data were used to support the findings of this study.

\section{Conflicts of Interest}

The authors declare that there are no conflicts of interest regarding the publication of this paper.

\section{Authors' Contributions}

The study was carried out in collaboration among all authors. All authors read and approved the final manuscript.

\section{Acknowledgments}

The authors were supported financially by the National Natural Science Foundation of China (11871302 and 11571296).

\section{References}

[1] I. Koponen, "Analytic approach to the problem of convergence of truncated Lévy flights towards the Gaussian stochastic process," Physical Review E, vol. 52, no. 1, pp. 1197-1199, 1995.

[2] A. Chakrabarty and M. M. Meerschaert, "Tempered stable laws as random walk limits," Statistics \& Probability Letters, vol. 81, no. 8, pp. 989-997, 2011.

[3] A. Kolmogorov, "Wiener spiral and some other interesting curves in Hilbert space," Dokl. Akad. Nauk SSSR, vol. 26, pp. 115-118, 1940.

[4] L. Leibenson, "General problem of the movement of a compressible fluid in a porous medium," Izv. Akad. Nauk Kirgiz, vol. 9, pp. 7-10, 1983, in Russian.

[5] F. Wang, L. Liu, and Y. Wu, "A numerical algorithm for a class of fractional BVPs with $p$-Laplacian operator and singularity-the convergence and dependence analysis," Applied Mathematics and Computation, vol. 382, Article ID 125339, 2020.

[6] J. Xu, J. Jiang, and D. O'Regan, "Positive solutions for a class of $p$-laplacian hadamard fractional-order three-point boundary value problems," Mathematics, vol. 8, no. 3, p. 308, 2020.

[7] F. Wang, L. Liu, Y. Wu, and Y. Zou, "Iterative analysis of the unique positive solution for a class of singular nonlinear boundary value problems involving two types of fractional derivatives with $p$-laplacian operator," Complexity, vol. 2019, Article ID 2319062, 21 pages, 2019.

[8] J. Jiang, D. O’Regan, J. Xu, and Y. Cui, "Positive solutions for a hadamard fractional p-laplacian three-point boundary value problem," Mathematics, vol. 7, no. 5, p. 439, 2019.

[9] X. Zhang, L. Liu, and Y. Wu, "The uniqueness of positive solution for a fractional order model of turbulent flow in a porous medium," Applied Mathematics Letters, vol. 37, pp. 26-33, 2014.

[10] L. Guo and L. Liu, "Unique iterative positive solutions for a singular $p$-laplacian fractional differential equation system with infinite-point boundary conditions," Boundary Value Problems, vol. 2019, no. 1, p. 113, 2019.

[11] M. Fečkan, M. Pospíšil, and J. Wang, "Note on weakly fractional differential equations," Advances in Difference Equations, vol. 2019, no. 1, p. 11, 2019.
[12] H. Liu and R. Xu, "Oscillation for a class of right fractional differential equations on the right half line with damping," Discrete Dynamics in Nature and Society, vol. 2019, Article ID 4902718, 9 pages, 2019.

[13] Z. You, M. Fečkan, and J. Wang, "Relative controllability of fractional delay differential equations via delayed perturbation of Mittag-Leffler functions," Journal of Computational and Applied Mathematics, vol. 378, Article ID 112939, 2020.

[14] D. Liu, K. Zhang, and K. Zhang, "Existence of positive solutions to a boundary value problem for a delayed singular high order fractional differential equation with signchanging nonlinearity," Journal of Applied Analysis \& Computation, vol. 10, no. 3, pp. 1073-1093, 2020.

[15] S. Zhai, C. Ye, and Z. Weng, "A fast and efficient numerical algorithm for fractional Allen-Cahn with precise nonlocal mass conservation," Applied Mathematics Letters, vol. 103, Article ID 106190, 2020.

[16] J. Zhang, J. Wang, and Y. Zhou, "Numerical analysis for Klein-Gordon equation with time-space fractional derivatives," Mathematical Methods in the Applied Sciences, vol. 43, no. 6, pp. 3689-3700, 2020.

[17] W. Guan, L.-P. Ma, D.-B. Wang, and J.-L. Zhang, "Infinitely many solutions for a class of sublinear fractional Schrödinger-Poisson systems," Quaestiones Mathematicae, vol. 2020, no. 1, pp. 1-11, 2020.

[18] Y. Wang, "The Greens function of a class of two-term fractional differential equation boundary value problem and its applications," Advances in Difference Equations, vol. 2020, no. 1, pp. 1-20, 2020.

[19] J. Zhang, J. Wang, and Y. Zhou, "Numerical analysis for time-fractional Schrödinger equation on two space dimensions," Advances in Difference Equations, vol. 2020, no. 1, p. 53, 2020.

[20] J. Mao, Z. Zhao, and C. Wang, "The unique iterative positive solution of fractional boundary value problem with q-difference," Applied Mathematics Letters, vol. 100, Article ID 106002, 2020.

[21] B. Zhang, W. Zhu, Y. Xia, and Y. Bai, "A unified analysis of exact traveling wave solutions for the fractional-order and integer-order Biswas-Milovic equation: via bifurcation theory of dynamical system," Qualitative Theory of Dynamical Systems, vol. 19, no. 1, p. 11, 2020.

[22] J. He, X. Zhang, L. Liu, Y. Wu, and Y. Cui, "A singular fractional Kelvin-Voigt model involving a nonlinear operator and their convergence properties," Boundary Value Problems, vol. 2019, p. 112, 2019.

[23] M. Fečkan, K. Marynets, and J. Wang, "Periodic boundary value problems for higher-order fractional differential systems," Mathematical Methods in the Applied Sciences, vol. 42, no. 10, pp. 3616-3632, 2019.

[24] K. Shah and J. Wang, "A numerical scheme based on nondiscretization of data for boundary value problems of fractional order differential equations," Revista de la Real Academia de Ciencias Exactas, Físicas y Naturales. Serie A. Matemáticas, vol. 113, no. 3, pp. 2277-2294, 2019.

[25] S. Song, B. Zhang, X. Song, Y. Zhang, Z. Zhang, and W. Li, "Fractional-order adaptive neuro-fuzzy sliding mode Ho control for fuzzy singularly perturbed systems," Journal of the Franklin Institute, vol. 356, no. 10, pp. 5027-5048, 2019.

[26] L. Ren, J. Wang, and D. O’Regan, “Asymptotically periodic behavior of solutions of fractional evolution equations of order $1<\alpha<2$," Mathematica Slovaca, vol. 69, no. 3, pp. 599-610, 2019. 
[27] Y. Wang, "Existence and multiplicity of positive solutions for a class of singular fractional nonlocal boundary value problems," Boundary Value Problems, vol. 2019, no. 1, pp. 1-18, 2019.

[28] J. Zhang, S. Lin, and J. Wang, "An efficient numerical approach to solve Schrödinger equations with space fractional derivative," Mathematical Methods in the Applied Sciences, vol. 42, no. 5, pp. 1596-1608, 2019.

[29] H. Wang and J. Jiang, "Multiple positive solutions to singular fractional differential equations with integral boundary conditions involving $p$ - $q$-order derivatives," Advances in Difference Equations, vol. 2020, no. 1, pp. 1-13, 2020.

[30] X. Zhang, L. Liu, and Y. Wu, "Variational structure and multiple solutions for a fractional advection-dispersion equation," Computers \& Mathematics with Applications, vol. 68, no. 12, pp. 1794-1805, 2014.

[31] B. Zhu, L. Liu, and Y. Wu, "Existence and uniqueness of global mild solutions for a class of nonlinear fractional reaction-diffusion equations with delay," Computers \& Mathematics with Applications, vol. 78, no. 6, pp. 1811-1818, 2019.

[32] J. Zhao, Y. Zhang, and Y. Xu, "Implicit Runge-Kutta and spectral Galerkin methods for Riesz space fractional/distributed-order diffusion equation," Computational and Applied Mathematics, vol. 39, no. 2, p. 47, 2020.

[33] X. Zhang, L. Liu, Y. Wu, and B. Wiwatanapataphee, "Nontrivial solutions for a fractional advection dispersion equation in anomalous diffusion," Applied Mathematics Letters, vol. 66, pp. 1-8, 2017.

[34] D. Ma, L. Liu, and Y. Wu, "Existence of nontrivial solutions for a system of fractional advection-dispersion equations," Revista de la Real Academia de Ciencias Exactas, Físicas y Naturales. Serie A. Matemáticas, vol. 113, no. 2, pp. 10411057, 2019.

[35] X. Zhang, C. Mao, L. Liu, and Y. Wu, "Exact iterative solution for an abstract fractional dynamic system model for Bioprocess," Qualitative Theory of Dynamical Systems, vol. 16, no. 1, pp. 205-222, 2017.

[36] Y. Wang, L. Liu, X. Zhang, and Y. Wu, "Positive solutions of an abstract fractional semipositone differential system model for bioprocesses of HIV infection," Applied Mathematics and Computation, vol. 258, pp. 312-324, 2015.

[37] M. Li and J. Wang, "Finite time stability and relative controllability of Riemann-Liouville fractional delay differential equations," Mathematical Methods in the Applied Sciences, vol. 42, no. 18, pp. 6607-6623, 2019.

[38] X. Zhang, L. Liu, Y. Wu, and Y. Cui, "New result on the critical exponent for solution of an ordinary fractional differential problem," Journal of Function Spaces, vol. 2017, Article ID 3976469, 4 pages, 2017.

[39] F. Wang, L. Liu, and Y. Wu, "Iterative unique positive solutions for a new class of nonlinear singular higher order fractional differential equations with mixed-type boundary value conditions," Journal of Inequalities and Applications, vol. 2019, no. 1, p. 210, 2019.

[40] X. Zhang, L. Liu, and Y. Wu, "Multiple positive solutions of a singular fractional differential equation with negatively perturbed term," Mathematical and Computer Modelling, vol. 55, no. 3-4, pp. 1263-1274, 2012.

[41] J. Wang, A. Zada, and H. Waheed, "Stability analysis of a coupled system of nonlinear implicit fractional anti-periodic boundary value problem," Mathematical Methods in the Applied Sciences, vol. 42, no. 18, pp. 6706-6732, 2019.
[42] X. Zhang, L. Liu, and Y. Wu, "The uniqueness of positive solution for a singular fractional differential system involving derivatives," Communications in Nonlinear Science and Numerical Simulation, vol. 18, no. 6, pp. 1400-1409, 2013.

[43] J. Zhao, Y. Zhang, and Y. Xu, "Implicit Runge-Kutta and spectral Galerkin methods for the two-dimensional nonlinear Riesz space fractional diffusion equation," Applied Mathematics and Computation, vol. 386, Article ID 125505, 2020.

[44] J. He, X. Zhang, L. Liu, Y. Wu, and Y. Cui, "Existence and asymptotic analysis of positive solutions for a singular fractional differential equation with nonlocal boundary conditions," Boundary Value Problems, vol. 2018, p. 189, 2018.

[45] J. Wu, X. Zhang, L. Liu, Y. Wu, and Y. Cui, “The convergence analysis and error estimation for unique solution of a $p$-Laplacian fractional differential equation with singular decreasing nonlinearity," Boundary Value Problems, vol. 2018, p. 82, 2018.

[46] J. Wu, X. Zhang, L. Liu, Y. Wu, and Y. Cui, "Convergence analysis of iterative scheme and error estimation of positive solution for a fractional differential equation," Mathematical Modelling and Analysis, vol. 23, no. 4, pp. 611-626, 2018.

[47] T. Ren, S. Li, X. Zhang, and L. Liu, "Maximum and minimum solutions for a nonlocal $p$-Laplacian fractional differential system from eco-economical processes," Boundary Value Problems, vol. 2017, no. 1, p. 118, 2017.

[48] J. Wu, X. Zhang, L. Liu, Y. Wu, and B. Wiwatanapataphee, "Iterated logarithm and estimation of solution for a fractional order differential equation," Boundary Value Problems, vol. 2016, p. 116, 2016.

[49] J. Wu, X. Zhang, L. Liu, and Y. Wu, "Twin iterative solutions for a fractional differential turbulent flow model," Boundary Value Problems, vol. 2016, p. 98, 2016.

[50] X. Zhang, L. Liu, Y. Wu, and B. Wiwatanapataphee, "The spectral analysis for a singular fractional differential equation with a signed measure," Applied Mathematics and Computation, vol. 257, pp. 252-263, 2015.

[51] Y. Wang, "Positive solutions for a class of two-term fractional differential equations with multipoint boundary value conditions," Advances in Difference Equations, vol. 2019, no. 1, p. 304, 2019.

[52] X. Zhang, L. Liu, and Y. Wu, "The eigenvalue problem for a singular higher order fractional differential equation involving fractional derivatives," Applied Mathematics and Computation, vol. 218, no. 17, pp. 8526-8536, 2012.

[53] D. Xu and F. Meng, "Oscillation criteria of certain fractional partial differential equations," Advances in Difference Equations, vol. 2019, no. 1, p. 460, 2019.

[54] X. Zhang, L. Liu, B. Wiwatanapataphee, and Y. Wu, "The eigenvalue for a class of singular $p$-Laplacian fractional differential equations involving the Riemann-Stieltjes integral boundary condition," Applied Mathematics and Computation, vol. 235, pp. 412-422, 2014.

[55] X. Hao, H. Sun, L. Liu, and D.-B. Wang, "Positive solutions for semipositone fractional integral boundary value problem on the half-line," Revista de la Real Academia de Ciencias Exactas, Físicas y Naturales. Serie A. Matemáticas, vol. 113, no. 4, pp. 3055-3067, 2019.

[56] X. Zhang, L. Liu, and Y. Wu, "Existence results for multiple positive solutions of nonlinear higher order perturbed fractional differential equations with derivatives," Applied Mathematics and Computation, vol. 219, no. 4, pp. 14201433, 2012. 
[57] J. Zhang, H. Chen, S. Lin, and J. Wang, "Finite difference/ spectral approximation for a time-space fractional equation on two and three space dimensions," Computers \& Mathematics with Applications, vol. 78, no. 6, pp. 1937-1946, 2019.

[58] D. Luo, J. Wang, and D. Shen, " $P D^{\alpha}$-type distributed learning control for nonlinear fractional order multiagent systems," Mathematical Methods in the Applied Sciences, vol. 42, no. 13, pp. 4543-4553, 2019.

[59] T. Ren, H. Xiao, Z. Zhou et al., "The iterative scheme and the convergence analysis of unique solution for a singular fractional differential equation from the eco-economic complex systems co-evolution process," Complexity, vol. 2019, Article ID 9278056, 15 pages, 2019.

[60] X. Zhang, L. Liu, Y. Wu, and Y. Lu, "The iterative solutions of nonlinear fractional differential equations," Applied Mathematics and Computation, vol. 219, no. 9, pp. 4680-4691, 2013.

[61] S. Zhang, Y. Wei, and B. Xu, "Fractional soliton dynamics and spectral transform of time-fractional nonlinear systems: a concrete example," Complexity, vol. 2019, no. 59, 9 pages, Article ID 7952871, 2019.

[62] H. Ming, J. Wang, and M. Fečkan, "The application of fractional calculus in Chinese economic growth models," Mathematics, vol. 7, no. 8, p. 665, 2019.

[63] W. Liu, L. Liu, and Y. Wu, "Existence of solutions for integral boundary value problems of singular Hadamard-type fractional differential equations on infinite interval," Advances in Difference Equations, vol. 2020, p. 274, 2020.

[64] Y. Sun and R. Xu, "New Hermite-Hadamard type inequalities for psi-Riemann-Liouville fractional integral via convex functions," Discrete Dynamics in Nature and Society, vol. 2020, Article ID 6953962, 17 pages, 2020.

[65] Y. Ding, J. Jiang, D. ORegan, and J. Xu, "Positive solutions for a system of Hadamard-type fractional differential equations with semipositone nonlinearities," Complexity, vol. 2020, Article ID 9742418, 14 pages, 2020.

[66] X. Wu, J. Wang, and A. J. Zhang, "Hermite-hadamard-type inequalities for convex functions via the fractional integrals with exponential kernel," Mathematics, vol. 7, no. 9, p. 845, 2019.

[67] P. Yang, J. Wang, and Y. Zhou, "Representation of solution for a linear fractional delay differential equation of Hadamard type," Advances in Difference Equations, vol. 2019, no. 1, p. 300, 2019.

[68] J. Jiang, D. O’Regan, J. Xu, and Z. Fu, “Positive solutions for a system of nonlinear Hadamard fractional differential equations involving coupled integral boundary conditions," Journal of Inequalities and Applications, vol. 2019, no. 1, pp. 1-18, 2019.

[69] J. Mao, Z. Zhao, and C. Wang, "The unique positive solution for singular Hadamard fractional boundary value problems," Journal of Function Spaces, vol. 2019, Article ID 923490, 6 pages, 2019.

[70] K. Liu, J. Wang, and D. ORegan, "On the Hermite-Hadamard type inequality for $\psi$-Riemann-Liouville fractional integrals via convex functions," Journal of Inequalities and Applications, vol. 2019, no. 1, p. 27, 2019.

[71] X. Zhang, L. Yu, J. Jiang, Y. Wu, and Y. Cui, "Positive solutions for a weakly singular Hadamard-type fractional differential equation with changing-sign nonlinearity," Journal of Function Spaces, vol. 2020, no. 62, 10 pages, Article ID 5623589, 2020.

[72] M. Ahmad, J. Jiang, A. Zada, S. Shah, and J. Xu, “Analysis of coupled system of implicit fractional differential equations involving Katugampola-Caputo fractional derivative," Complexcity, vol. 2020, Article ID 9285686, 11 pages, 2020.

[73] H. Liu and R. Xu, "The oscillatory of linear conformable fractional differential equations of Kamenev type," Dynamics in Nature and Society, vol. 2020, Article ID 3857592, 8 pages, 2020.

[74] Z. Zheng, H. Liu, J. Cai, and Y. Zhang, "Criteria of limit point case for conformable fractional Sturm Liouville operators," Mathematical Methods in the Applied Sciences, vol. 43, no. 5, pp. 2548-2557, 2020.

[75] X. Wang, J. Wang, and M. Fečkan, "Controllability of conformable differential systems," Nonlinear Analysis: Modelling and Control, vol. 25, no. 4, pp. 658-674, 2020.

[76] J. Shao and Z. Zheng, "Kamenev type oscillatory criteria for linear conformable fractional differential equations," Discrete Dynamics in Nature and Society, vol. 2019, Article ID 2310185, 10 pages, 2019.

[77] K. Liu, M. Fečkan, and J. Wang, “A fixed-point approach to the Hyers-Ulam stability of Caputo-Fabrizio fractional differential equations," Mathematics, vol. 8, no. 4, p. 647, 2020.

[78] K. Liu, M. Fečkan, D. O’Regan, and J. Wang, "Hyers-ulam stability and existence of solutions for differential equations with caputo-fabrizio fractional derivative," Mathematics, vol. 7, no. 4, p. 333, 2019

[79] J. Wang, A. G. Ibrahim, and D. O’Regan, “Finite approximate controllability of Hilfer fractional semilinear differential equations," Miskolc Mathematical Notes, vol. 21, no. 1, pp. 489-507, 2020.

[80] K. Liu, J. Wang, and D. O’Regan, “Ulam-Hyers-MittagLeffler stability for $\psi$-Hilfer fractional-order delay differential equations," Advances in Difference Equations, vol. 2019, no. 1, pp. 1-12, 2019.

[81] J. Wang, X. Liu, and D. O’Regan, “On the approximate controllability for Hilfer fractional evolution hemivariational inequalities," Numerical Functional Analysis and Optimization, vol. 40, no. 7, pp. 743-762, 2019.

[82] J. Wang, G. Ibrahim, and D. D. ORegan, "Controllability of Hilfer fractional noninstantaneous impulsive semilinear differential inclusions with nonlocal conditions," Nonlinear Analysis: Modelling and Control, vol. 24, no. 6, pp. 958-984, 2019.

[83] B. Zhou, L. Zhang, G. Xing, and N. Zhang, "Existenceuniqueness and monotone iteration of positive solutions to nonlinear tempered fractional differential equation with p-Laplacian operator," Boundary Value Problems, vol. 2020, no. 1, p. 117, 2020.

[84] L. Ren, J. Wang, J. Wang, and M. Fečkan, "Periodic mild solutions of impulsive fractional evolution equations," AIMS Mathematics, vol. 5, no. 1, pp. 497-506, 2020.

[85] B. Zhang, Y. Xia, L. Zhu, H. Liu, and L. Gu, "Global stability of fractional order coupled systems with impulses via a graphic approach," Mathematics, vol. 7, no. 8, p. 744, 2019.

[86] J. Wang, A. G. Ibrahim, and D. O’Regan, “Global attracting solutions to Hilfer fractional differential inclusions of Sobolev type with noninstantaneous impulses and nonlocal conditions," Nonlinear Analysis: Modelling and Control, vol. 24 , no. 5, pp. 775-803, 2019.

[87] J. Wang and M. Feckan, "Periodic solutions and stability of linear evolution equations with noninstantaneous impulses," Miskolc Mathematical Notes, vol. 20, no. 2, pp. 1299-1313, 2019.

[88] Y. Chen and J. Wang, "Continuous dependence of solutions of integer and fractional order non-instantaneous impulsive 
equations with random impulsive and junction points," Mathematics, vol. 7, no. 4, p. 331, 2019.

[89] J. Wang, A. G. Ibrahim, and D. O’Regan, "Nonemptyness and compactness of the solution set for fractional evolution inclusions with non-instantaneous impulses," Electronic Journal of Differential Equations, vol. 2019, no. 37, pp. 1-17, 2019.

[90] S. Xu, H. Lv, H. Liu, and A. Liu, "Robust control of disturbed fractional-order economical chaotic systems with uncertain parameters," Complexity, vol. 2019, Article ID 7567695, 13 pages, 2019.

[91] M. Fečkan, T. Sathiyaraj, and J. Wang, "Synchronization of butterfly fractional order chaotic system," Mathematics, vol. 8, no. 3, p. 446, 2020.

[92] F. Wang and Z. Zheng, "Quasi-projective synchronization of fractional order chaotic systems under input saturation," Physica A: Statistical Mechanics and Its Applications, vol. 534, Article ID 122132, 2019.

[93] S. Ha, H. Liu, S. Li, and A. Liu, "Backstepping-based adaptive fuzzy synchronization control for a class of fractional-order chaotic systems with input saturation," International Journal of Fuzzy Systems, vol. 21, no. 5, pp. 1571-1584, 2019.

[94] Y. Wang and H. Wang, "Triple positive solutions for fractional differential equation boundary value problems at resonance," Applied Mathematics Letters, vol. 106, Article ID 106376, 2020.

[95] Y. Wang and H. Wang, "Existence and nonexistence of positive solutions for high-order fractional differential equation boundary value problems at resonance," Journal of Function Spaces, vol. 2020, Article ID 2395238, 10 pages, 2020.

[96] Y. Wang, "Necessary conditions for the existence of positive solutions to fractional boundary value problems at resonance," Applied Mathematics Letters, vol. 97, pp. 34-40, 2019.

[97] X. Zhang, L. Liu, Y. Wu, and Y. Cui, “A sufficient and necessary condition of existence of blow-up radial solutions for a k-Hessian equation with a nonlinear operator," Nonlinear Analysis: Modelling and Control, vol. 25, no. 1, pp. 126-143, 2020.

[98] X. Zhang, J. Xu, J. Jiang, Y. Wu, and Y. Cui, “The convergence analysis and uniqueness of blow-up solutions for a Dirichlet problem of the general k-Hessian equations," Applied Mathematics Letters, vol. 102, Article ID 106018, 2020.

[99] X. Zhang, J. Jiang, Y. Wu, and Y. Cui, "The existence and nonexistence of entire large solutions for a quasilinear Schrodinger elliptic system by dual approach," Applied Mathematics Letters, vol. 100, Article ID 106018, 2020.

[100] X. Zhang, Y. Wu, and Y. Cui, "Existence and nonexistence of blow-up solutions for a Schrodinger equation involving a nonlinear operator," Applied Mathematics Letters, vol. 82, pp. 85-91, 2018.

[101] A. A. Kilbas, H. M. Srivastava, and J. J. Trujillo, "Theory and applications of fractional differential equations," in NorthHolland Mathematics Studies, pp. 1-523, Elsevier, Amsterdam, Netherlands, 2006. 